\title{
COMPUTATION PROGRAMS OF THE ASTRONOMICAL VESSEL POSITION
}

\author{
Chih-Li Chen \\ Department of Merchant Marine, National Taiwan Ocean University, Keelung, Taiwan, R.O.C., clchen@mail.ntou.edu.tw \\ Tsung-Hsuan Hsieh \\ Department of Merchant Marine, National Taiwan Ocean University, Keelung, Taiwan, R.O.C.
}

Follow this and additional works at: https://jmstt.ntou.edu.tw/journal

Part of the Computer Sciences Commons

\section{Recommended Citation}

Chen, Chih-Li and Hsieh, Tsung-Hsuan (2011) "COMPUTATION PROGRAMS OF THE ASTRONOMICAL VESSEL POSITION," Journal of Marine Science and Technology. Vol. 19: Iss. 1, Article 5.

DOI: $10.51400 / 2709-6998.2135$

Available at: https://jmstt.ntou.edu.tw/journal/vol19/iss1/5

This Research Article is brought to you for free and open access by Journal of Marine Science and Technology. It has been accepted for inclusion in Journal of Marine Science and Technology by an authorized editor of Journal of Marine Science and Technology. 


\section{COMPUTATION PROGRAMS OF THE ASTRONOMICAL VESSEL POSITION}

\section{Acknowledgements}

The first author would like to thank the National Science Council, Taiwan for financial support under contract number: NSC 96-2628-E-019-022-MY3. 


\title{
COMPUTATION PROGRAMS OF THE ASTRONOMICAL VESSEL POSITION
}

\author{
Chih-Li Chen* and Tsung-Hsuan Hsieh*
}

Key words: intercept method, observed altitude, astronomical vessel position.

\begin{abstract}
In open sea sailing, as opposed to sailing with satellite navigation systems, the astronomical vessel position (AVP) is not limited by military codes. If programs can be developed to solve the AVP directly, drawbacks of current methods for performing marine operations can be greatly improved. Complete AVP computations have two necessary points: compute the observed altitude and compute the AVP for two bodies. Thus, this paper utilizes Matlab ${ }^{\circledR}$ programming language to develop the observed altitude (ObsAltPro) and the AVP by using intercept method (IMPro-2) programs, respectively. Adopting the ObsAltPro can avoid the use of nautical almanacs and directly obtain the observed altitudes of various celestial bodies between 1986 and 2050. As for merits of using the IMPro-2, they can skip limits of tabular methods and directly use the dead reckoning (DR) as the input variable to replace the assumed position (AP). To address the deficiencies of the intercept method (IM), iteration computation is added into the latter program as a verification function. The above two programs, through demonstrated examples, have shown advantages of being simple, fast, and accurate.
\end{abstract}

\section{INTRODUCTION}

For ocean navigation, the main approaches of positioning are the classical method of astronomical vessel position (AVP) and advanced satellite navigation systems. Due to the complicated, tedious and time consuming nature of current AVP and the accuracy limitations due to tabular methods, the majority of navigators prefer using satellite navigation systems. However, the AVP has the advantage of not being limited by military codes. If assisted by computerized programs, the practical operation process can be greatly improved. Thus, in searching for computation software for the AVP, it was discovered that the software developed by the U.S. Navy is not purchasable [5].

Paper submitted 08/05/09; revised 12/18/09; accepted 12/23/09. Author for correspondence: Chih-Li Chen (e-mail: clchen@mail.ntou.edu.tw).

*Department of Merchant Marine, National Taiwan Ocean University, Keelung, Taiwan, R.O.C.
Other softwares, NavPac developed by a British research organization [7], Navigator [12] and STARPILOT PC [13] developed by private companies, use the intercept method (IM). However, they do not have the verification function of iteration computation. This disparity between expectations and reality leads to the motivation of this article.

The task in developing the AVP computational programs is to understand the process. Due to the complicated astronomical algorithms that are involved with nautical almanacs, this paper will not discuss this topic in detail. Other than that, as the IM is the main way of the sight reduction methods, which requires more than two lines of position (LOPs) to obtain AVP this paper will develop the programs aiming at "the observed altitude of various celestial bodies," namely, ObsAltPro and "the intercept method for solving AVP for two bodies condition," namely, IMPro-2, respectively as shown in Fig. 1.

Apart from this introduction, the following sections are arranged as follows. Sections II organizes the computation procedures of ObsAltPro and IMPro-2. Based on this, sections III uses MATLAB ${ }^{\circledR}$ to develop the programs. Sections IV then verifies the self-developed programs through demonstrated examples. Finally, sections V provides some concrete conclusions.

\section{ORGANIZING COMPUTATION PROCEDURES}

\section{Computation Procedures of Observed Altitude from Sextant Altitude}

This paper uses the nautical almanac tabular formulae [6] to establish the computation procedures in conjunction with the semi-diameter $(S D)$ of Sun and the horizontal parallaxes $(H P)$ of the Moon, Venus, and Mars obtained from the works of Jean Meeus published in 1998 [2, 3, 10]. These formulae are only suitable for use between 1986 and 2050. The organized computation procedures are as follows:

Step 1: Calculate the dip of the horizon (Dip).

$$
\text { Dip }=0.0293^{\circ} \times \sqrt{h e},
$$

where he is the height of eye above the horizon in meters. 


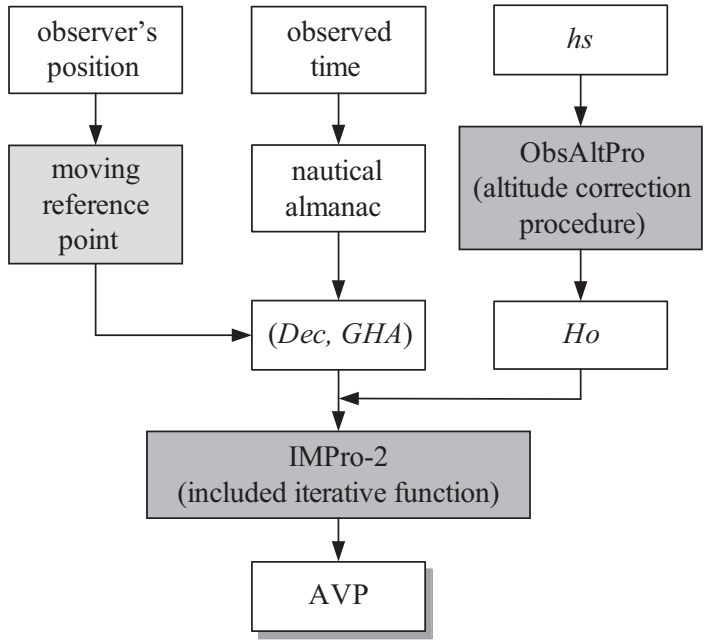

Fig. 1. Computational programs of the AVP.

Step 2: Calculate the apparent altitude $(h a)$ of the observed body.

$$
h a=h s \pm I \pm I C-D i p,
$$

where $h s$ is the sextant altitude, $I$ is the sextant instrument correction, and $I C$ is the sextant index correction.

Step 3: Calculate refraction $(R)$.

- The refraction formula at a standard temperature of $10^{\circ}$ Celsius (C) and standard pressure of 1010 millibars (mb) is as follows:

$$
R_{O}=\frac{0.0167^{\circ}}{\tan \left[h a+\left(\frac{7.32}{h a+4.32}\right)\right]},
$$

in which $R_{O}$ represents refraction for standard condition.

- If the temperature $T^{\circ} \mathrm{C}$ and pressure $B \mathrm{mb}$ are known, then the refraction formula is as follows:

$$
R=\left(\frac{0.28 \times B}{T+273}\right) \times R_{O},
$$

in which $R$ represents refraction for non-standard atmospheric conditions.

Step 4: Calculate semi-diameter $(S D)$.

- Sun: Computing the distance between the Sun and the Earth using the VSOP 87 method, namely obtaining the radius vector of the Sun $(\Delta s)$, and then further calculating the Sun semi-diameter in Eq. (5) [2].

$$
S D=\frac{959.63^{\prime \prime}}{\Delta s},
$$

where $\Delta s$ denotes the Sun radius vector in astronomical units (AU), and the Sun $S D$ in arc minutes.

- Moon: Computing the distance between the Moon and the Earth using the ELP2000/82 method can obtain the radius vector of the Moon $(\Delta m)$. Then, the Moon $H P$ is calculated in Eq. (6) [3], and substituting the HP into Eq. (7) yields the Moon semi-diameter $(S D)$,

$$
\begin{aligned}
& \sin H P=\frac{6378.14}{\Delta m}, \\
& S D=0.2724^{\circ} \times H P .
\end{aligned}
$$

Where $\Delta m$ denotes the Moon radius vector in kilometers, the Moon $H P$ in degrees, and the Moon $S D$ in arc minutes.

- Navigational stars and planets: the $S D$ is ignored due to the center of their observed bodies is easier to determine.

Step 5: Calculate the parallax $(P)$, from the $H P$ and the $h a$, the formula is as follows:

$$
P=H P \times \cos (h a)
$$

- Sun: Substituting the Sun $H P 0.0024^{\circ}$ in Eq. (8) to calculate the Sun $P$.

- Moon: After obtaining the Moon HP through Eq. (6), substituting the $H P$ into Eq. (8) yields the Moon $P$.

- Venus and Mars: After computing the distances between Venus and the Earth, Mars and the Earth, with the VSOP 87 method, respectively, one can obtain the radius vector of Venus or Mars $(\Delta s t)$. Then, the $H P$ is calculated in Eq. (9) [3]. Substituting the $H P$ into Eq. (8) yields the $P$ of Venus or Mars.

$$
\sin H P=\frac{\sin 8.794^{\prime \prime}}{\Delta s t}
$$

where $\Delta s t$ denotes the radius vector of Venus or Mars in kilometers, and the Venus or Mars HP in degrees.

- Jupiter, Saturn, and navigational stars: the $P$ is ignored due to their far distance from the Earth.

Step 6: Calculate the observed altitude $(\mathrm{Ho})$.

$$
H o=h a-R \pm S D+P,
$$

where Ho represents observed altitude.

\section{Computation Procedures of AVP by Using the Intercept Method for Two Bodies}

The basic idea of the IM is to choose the assumed position (AP) at the nearby the dead reckoning (DR) position, and take it as the reference point to calculate the altitude and azimuth. By comparing the computed altitude with observed altitude, 


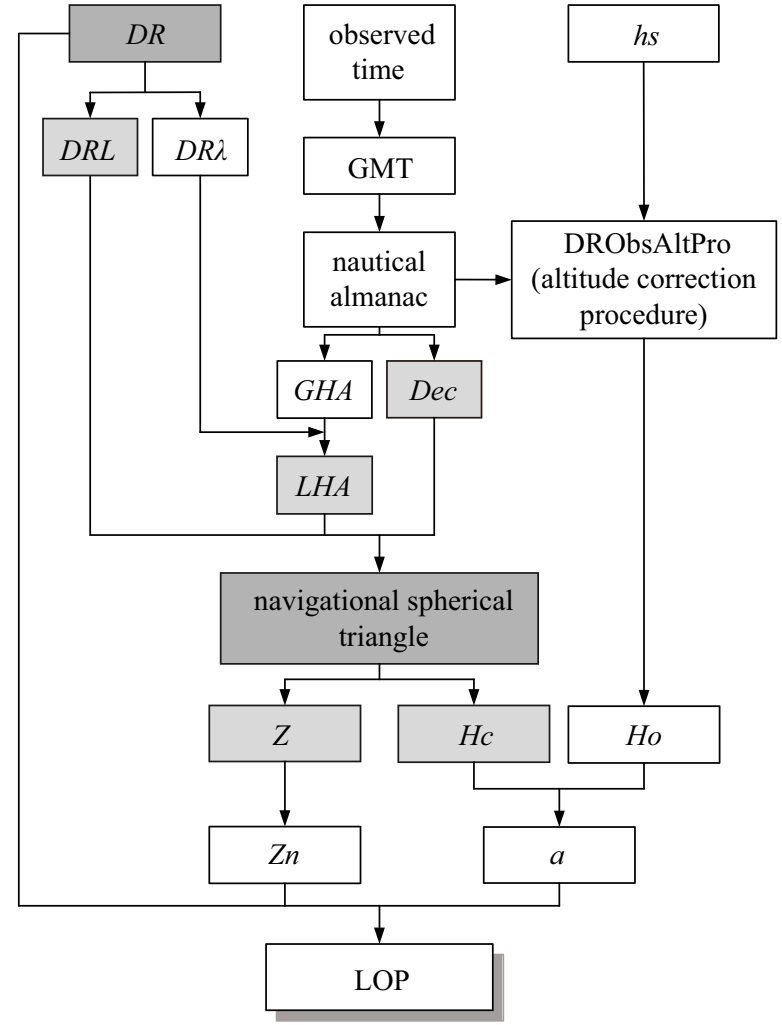

Fig. 2. The flowchart for solving the LOP by using the intercept method.

the difference of two altitudes (called the intercept, $a$ ) can be obtained. Therefore, once the AP, the computed azimuth angle $(Z)$ of the body, and the intercept are all determined, the LOP can be plotted according to the three elements. Hence, the IM is essentially a kind of trial-and-error method. If the computation method is adopted, the choice of the initial position, such as the DR position in place of the AP, can be unconstrained. The flowchart for solving the LOP by using the IM is shown in Fig. 2. It should be note that an observed body's position variables in celestial equator coordinate, such as the declination (Dec) and the Greenwich hour angle (GHA), can be obtained at observed time by using the nautical almanac or related software. Besides, a celestial body's local hour angle (LHA) is the algebraic sum of its $G H A$ and the observer's longitude $(\lambda)$.

Computing an observed body's position variables in celestial horizon coordinate, such as the computed altitude $(H c)$ and azimuth angle $(Z)$, the IM is a navigational spherical triangle problem. That is, when the two sides (co-latitude and polar distance) and the included angle ( $L H A)$ of an oblique spherical triangle are given, the problem is to solve the third side (co-altitude) and the outer angle $(Z)$. When the simplicity and minimum error propagation are taken into account in the computation, the optimal formulae are the side cosine and the four-parts formula $[8,9]$. The formulae are as follows:

$\sin H c=\sin (D R L) \cdot \sin (D e c)+\cos (D R L) \cdot \cos (D e c) \cdot \cos L H A$, $\tan Z=\frac{\sin L H A}{[\cos (D R L) \cdot \tan (D e c)]-[\sin (D R L) \cdot \cos L H A]}$,

in which $D R L$ is the DR latitude, Dec is declination, $L H A$ is local hour angle, $H c$ is computed altitude, and $Z$ is azimuth angle.

Some important issues should be noted here. The first is that in the above equations if sign of $D e c$ is the same as $D R L$, it is treated as a positive quantity. On the contrary, it is treated as a negative quantity. The second is that the side cosine formula can be reduced to Eq. (11) because sine functions are odd and cosine functions are even. In addition, if the computation method is used, the accuracy of the obtained AVP can be increased and verified by the iteration computation $[4,8]$.

Basically, the classical problem of celestial navigation, in its simplest form, is the determination of an AVP; one intersection is resulted from the different LOPs of two celestial bodies, observed at known GMT on a known date. However, the most case is observing altitudes of the same or different celestial bodies at different time [14]. Thus, the running fix concept is usually adopted and after the course, speed, and period of two observing times have been identified. To this point, for complete computation procedure, the "moving reference point," should be taken into consideration. This is explicated below.

In order to move the circle of position (COP) or LOP to the same time, the running fix concept to do so is to use the rhumb line sailing in conjunction with the moving reference point, the geographical position (GP) of the COP or the DR of the LOP, is adopted $[1,5,8]$. The reason for obtaining a celestial fix is that each would have to be advanced or retired to the desired time for celestial fix, and making proper allowance for the travel of the vessel during the intervening time. The computation procedures are as follows:

Step 1: Calculate the moving distance $(d)$ and difference of latitude $(l)$ by using

$$
d=s \times t,
$$

and

$$
l=d \times \cos C,
$$

where $s$ is speed, $t$ is time interval, and $C$ is course angle.

Step 2: Calculate the new reference point latitude $\left(L_{2}\right)$ by using

$$
L_{2}=L_{1} \pm l
$$

where $L_{1}$ is the reference point latitude.

Step 3: Calculate the meridional difference $(m)$ by using 


$$
\begin{aligned}
& M L_{1}=a \ln \left[\tan \left(45^{\circ}+\frac{L_{1}}{2}\right) \times\left(\frac{1-e \sin L_{1}}{1+e \sin L_{1}}\right)^{\frac{e}{2}}\right], \\
& M L_{2}=a \ln \left[\tan \left(45^{\circ}+\frac{L_{2}}{2}\right) \times\left(\frac{1-e \sin L_{2}}{1+e \sin L_{2}}\right)^{\frac{e}{2}}\right],
\end{aligned}
$$

and

$$
m=M L_{1} \sim M L_{2},
$$

where $a$ is the equator's arc minute length, $e$ is earth flattening, $M L_{1}$ is the meridional parts at $L_{1}, M L_{2}$ is the meridional parts at $L_{2}$.

Step 4: Calculate the new reference point longitude $\left(\lambda_{2}\right)$ by using

$$
\begin{gathered}
D L o=m \times \tan C, \\
\lambda_{2}=\lambda_{1} \pm D L o,
\end{gathered}
$$

where $D L o$ is difference of longitude, and $\lambda_{1}$ is the reference point longitude.

However, as Eq. (17) can be seen, when the course angle is $90^{\circ}, \tan C=\infty$, and the $D L o$ can not be calculated. In this case, the parallel sailing should be used for calculating the DLo. The formula is as follows:

$$
D L o=d \times \sec L,
$$

where $L$ is the reference point latitude.

It is worth mentioning, that because of an irregular oblate spheroid of the earth, parameters of meridional parts are taken from the 1984 world geodetic system (WGS-84) [11], in which $a=\frac{21600^{\prime}}{2 \pi}, e=\sqrt{2 f-f^{2}}$, and $f=\frac{1}{298.257223563}$,

The parameters of the Global Positioning System (GPS) and Electronic Chart Display and Information System (ECDIS) both adopt WGS-84; therefore the applications of developed program in this paper are quite broad.

\section{DEVELOPING COMPUTATION PROGRAMS}

As explained in Sections II, this paper uses computation procedures of observed altitude from sextant altitude as the basis in developing the observed altitude program of various celestial bodies between 1986 and 2050, named ObsAltPro and its flowchart is shown in Fig. 3. As for computation procedures of the AVP, the IM method is used as the basis to

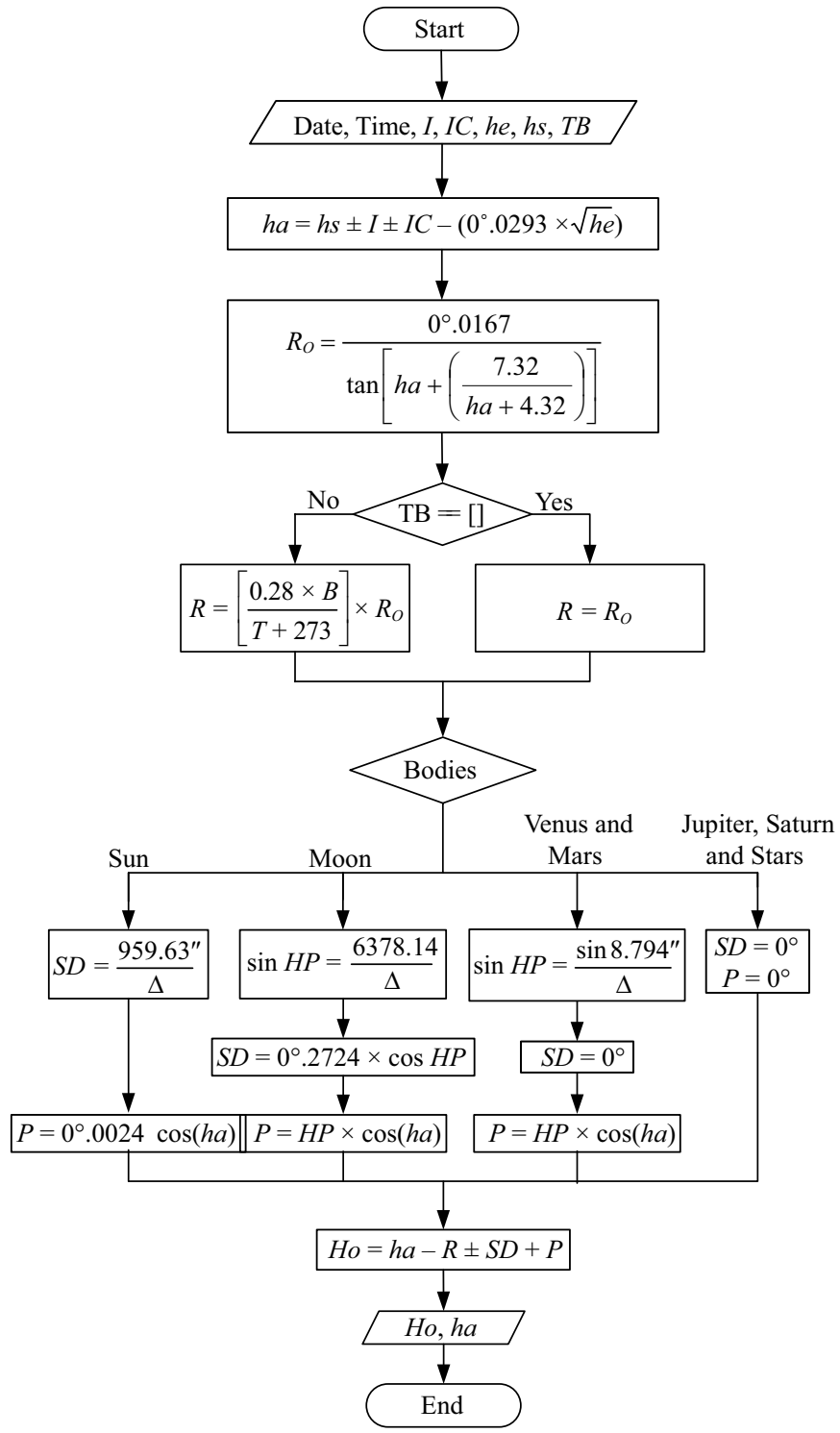

Fig. 3. The flowchart of the ObsAltPro program.

develop the IM program for two bodies, named IMPro-2, and the iteration computation function is provided for the accuracy of the AVP shown in Fig. 4. Moreover, to ensure the completion of the IMPro-2, moving reference point computation is included as shown in Fig. 5. For friendly use, the above two computation programs were both developed using the graphical user interface (GUI) of the MATLAB ${ }^{\circledR}$ programming language.

\section{DEMONSTRATED EXAMPLES}

Example 1: At 10-00-00 GMT (07-00-00 local time), June 16, 1994, the navigator obtains a sight of the Moon's upper limb. The sextant altitude $(h s)$ is $20^{\circ} 6.7^{\prime}$. Height of eye is 18 feet; there is no index error. Determine the observed altitude $(\mathrm{Ho})$ of the Moon [1]. 


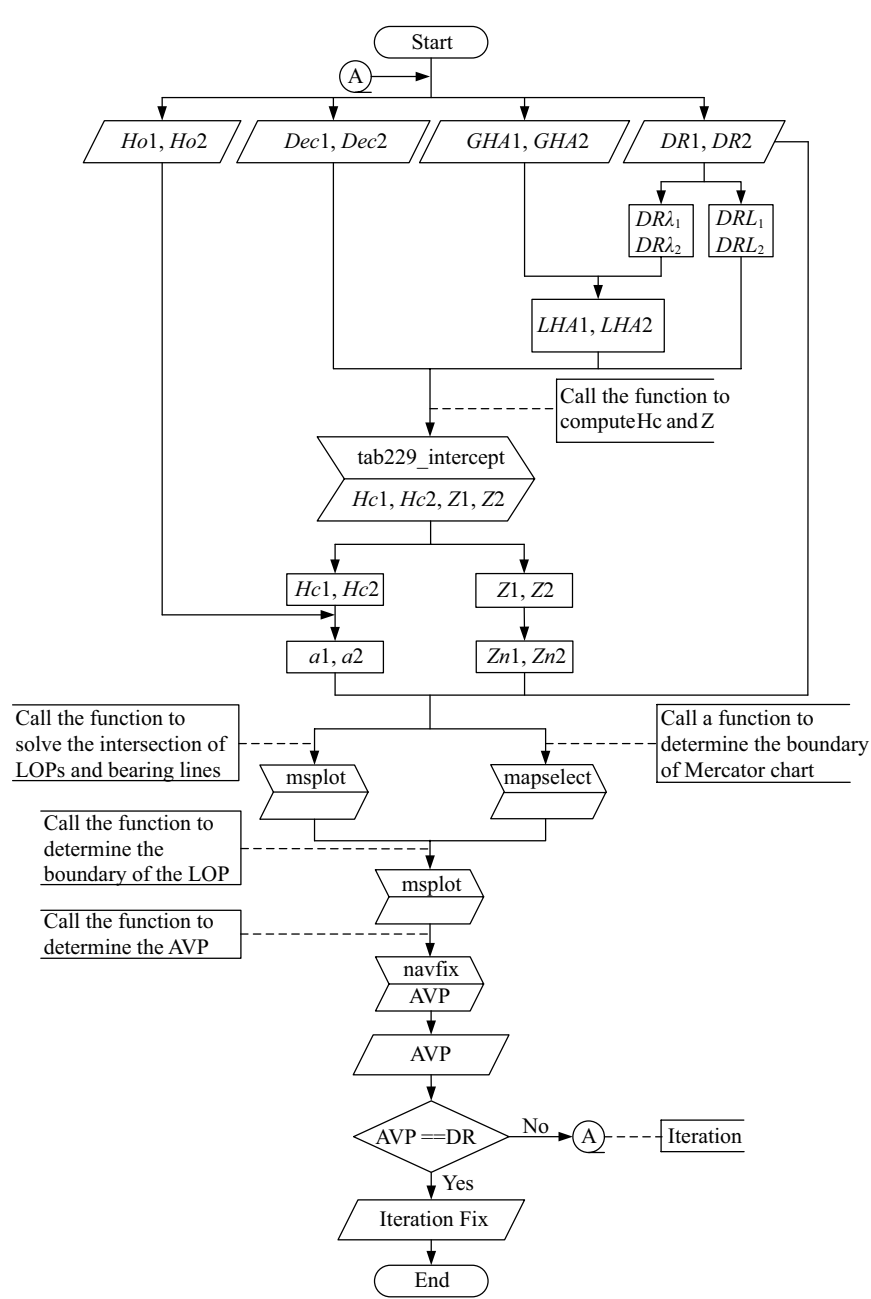

Fig. 4. The flowchart of the IMPro-2 program.

Required: The observed altitude can be determined in the following two approaches for sight reduction.

- Using the nautical almanac to solve the observed altitude.

- Using developed program ObsAltPro to solve the observed altitude directly.

\section{Solution:}

- Extracted from American Practical Navigator (No. 9), result of the Moon observed altitude is $26^{\circ} 37.1^{\prime}$ [1].

- Executing ObsAltPro shows the Moon observed altitude to be $26^{\circ} 37.1^{\prime}$, as shown in Fig. 6.

Explanation: For the same results, this example is verified that self-developed program is correct. Moreover, the ObsAltPro program, directly performs calculations using formulae, adopts GUI expression to demonstrate the advantages of being simple, fast and accurate. Besides, computerized program is more versatile than tabular method due to its design. That is, the ObsAltPro program does not require the use of nautical almanacs, and using this program can directly obtain the observed altitude of various celestial bodies between 1986 and 2050 .

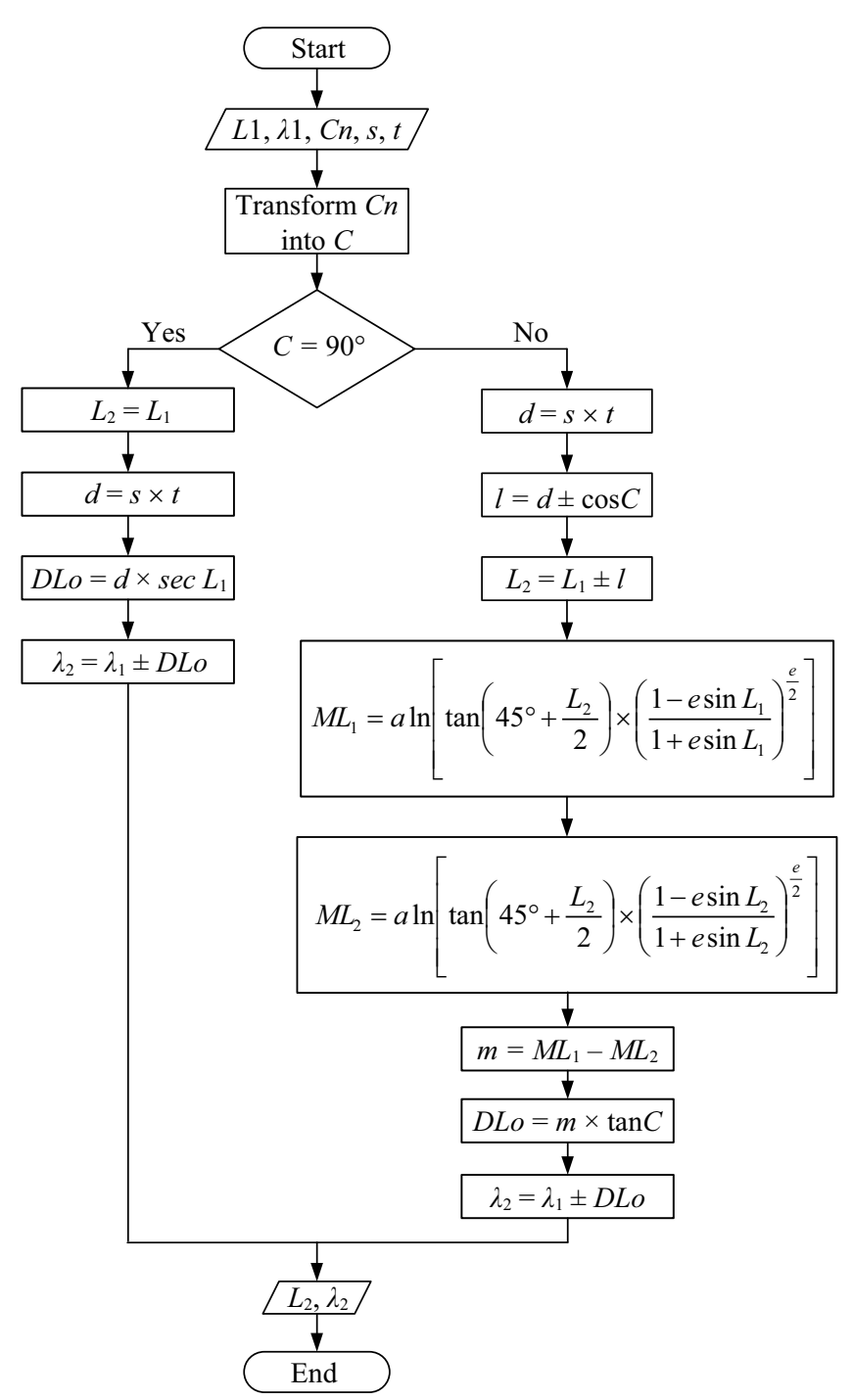

Fig. 5. The flowchart of moving reference point function in IMPro-2.

Example 2: On June 16, 1994, at 05-15-23 local time, at DR position $L 30^{\circ} \mathrm{N}, \lambda 045^{\circ} \mathrm{W}$, the navigator already took a sight of the Sun's upper limb. The ship is keeping on course $030^{\circ}$ and speed 10 knots. Again, at 07-00-00 local time, another sight of the Moon was obtained, as explicated in Example 1. Thus, the navigator wants to adopt the running fix concept to determine 0700 celestial fix, namely AVP. Therefore, the navigator records the needed information and further reduces it from the nautical almanac for sight reduction as shown in Table 1 [1].

Required: The AVP can be determined by using self-developed program, IMPro-2, to solve the AVP.

Solution: First, use the moving reference point function of IMPro-2 to obtain the post-moving reference position $L$ $30^{\circ} 15.1^{\prime} N, \lambda 044^{\circ} 50.0^{\prime} W$, as shown in the upper-left of Fig. 7. Then, treat the obtained position as the DR position of the panel, and enter the needed information, such as the Dec, 
Table 1. Extract of relevant information for sight reduction in example 2.

\begin{tabular}{ccccc}
\hline Body & ZT & $H o$ & Dec & GHA \\
\hline Sun & $05-15-23$ & $2^{\circ} 48.1^{\prime}$ & $23^{\circ} 20.5^{\prime} N$ & $303^{\circ} 42.1^{\prime}$ \\
Moon & $07-00-00$ & $26^{\circ} 37.1^{\prime}$ & $0^{\circ} 13.8^{\prime} N$ & $346^{\circ} 15.0^{\prime}$ \\
\hline
\end{tabular}

Source: [1] The American Practical Navigator, pp. 303-307 (2002).

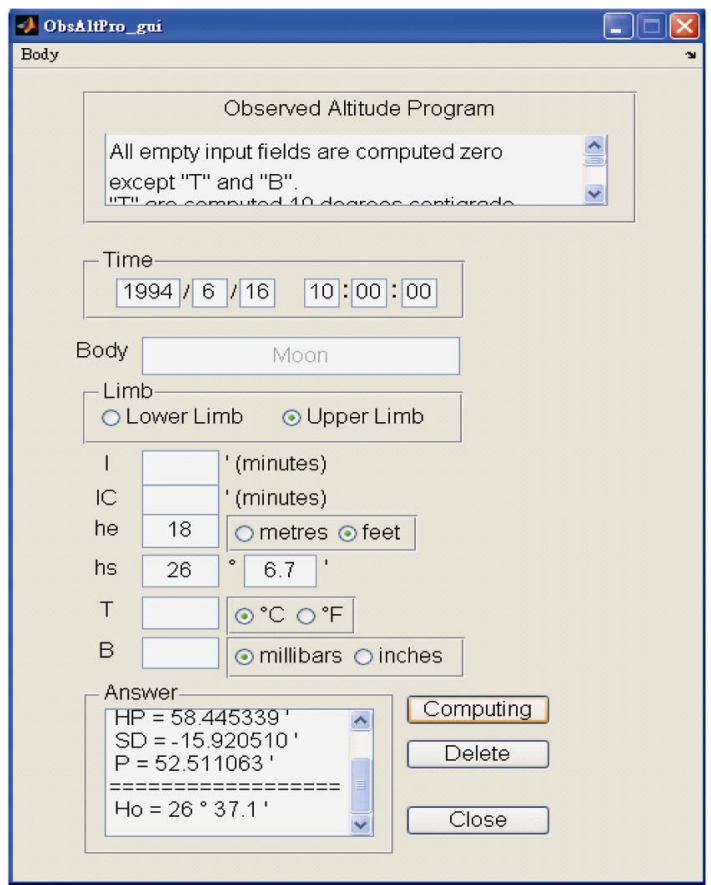

Fig. 6. Using the ObsAltPro to solve the observed altitude in example 1.

$G H A$, and $H o$, of the two celestial bodies (The Sun and the Moon), as shown in the lower-left of Fig. 7. Clicking the computing function can obtain an AVP of $L 30^{\circ} 48.4^{\prime} N, \lambda$ $044^{\circ} 58.0^{\prime} \mathrm{W}$. Further, clicking the iteration function can yield the accurate AVP of $L 30^{\circ} 48.5^{\prime} N, \lambda 044^{\circ} 58.0^{\prime} \mathrm{W}$, as shown in the lower-right of Fig. 7.

Explanation: This designed example is only demonstrated how to use IMPro-2 to solve the AVP.

Example 3: The $2004 \mathrm{DR}$ position of a vessel is $L 41^{\circ} 34.8^{\prime} \mathrm{N}$, $\lambda 017^{\circ} 00.5^{\prime} W$. At $20-03-58$, the star Capella is observed with a sextant. At 20-02-56, shortly before the above observation, another star, the Alkaid is spotted. The navigator records the needed information and further reduces it from the nautical almanac for sight reduction as shown in Table 2 [5].

Required: The AVP can be determined in the following three approaches for sight reduction.

- Using the IM in conjunction with the inspection tables (No. 229) to solve the AP, Zn and $a$, and plot the LOP. (Tabular method)
Table 2. Extract of relevant information for sight reduction in example 3.

\begin{tabular}{cccc}
\hline Body & ZT & Ho & \multicolumn{1}{c}{ GP } \\
\hline Alkaid & $20-03-06$ & $77^{\circ} 34.9^{\prime}$ & $\left(\begin{array}{l}49^{\circ} 25.7^{\prime} N \\
003^{\circ} 14.2^{\prime}\end{array}\right.$ \\
Capella & $20-04-08$ & $15^{\circ} 19.3^{\prime}$ & $\left(\begin{array}{l}45^{\circ} 58.4^{\prime} N \\
131^{\circ} 24.8^{\prime}\end{array}\right.$ \\
\hline
\end{tabular}

Source: [5] Dutton's Nautical Navigation, pp. 332-335 (2004).

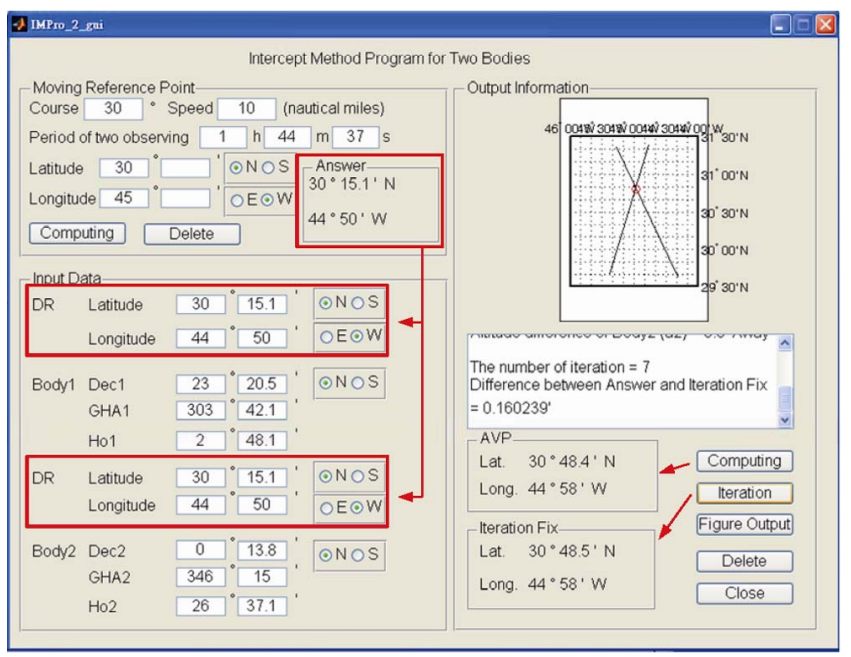

Fig. 7. Using the IMPro-2 to solve the AVP in example 2.

- Treating AP as the initial reference point, using developed program IMPro-2 to solve the possible AVP and verify it. (AP to run IMPro-2)

- Treating DR as the initial reference point, using developed program IMPro-2 to solve the possible AVP and verify it. (DR to run IMPro-2)

\section{Solution:}

- Extracted from Dutton's Nautical Navigation, results of AP, $Z n$ and $a$ are listed in Table 3 and the graphical AVP is $L 41^{\circ} 38.6^{\prime} \mathrm{N}, \lambda 017^{\circ} 08.1^{\prime} \mathrm{W}[5]$.

- AP was treated to run IMPro-2, AVP is $L 41^{\circ} 39.0^{\prime} N, \lambda$ $017^{\circ} 07.6^{\prime} W$. Then, executing the iteration function yields fixed AVP is $L 41^{\circ} 39.1^{\prime} N, \lambda 017^{\circ} 07.3^{\prime} \mathrm{W}$, as shown in Fig. 8 .

- DR was treated to run IMPro-2, AVP is $L 41^{\circ} 39.1^{\prime} N, \lambda$ $017^{\circ} 07.3^{\prime} W$. Then, executing the iteration function yields fixed AVP is $L 41^{\circ} 39.1^{\prime} N, \lambda 017^{\circ} 07.3^{\prime} \mathrm{W}$, as shown in Fig. 9.

Discussion: In actuality, this example is validated and the accurate AVP is pointed at $L 41^{\circ} 39.1^{\prime} N, \lambda 017^{\circ} 07.3^{\prime} \mathrm{W}$ from the paper of Hsu, T.P. et al. [8]. Consequently, it is demonstrated that DR running IMPro-2 can obtain the true AVP, more accurate than tabular method or AP running IMPro- 2 . However, executing the iteration function showed that, regardless of whether AP running IMPro-2 or DR running 
Table 3. Three elements for plotting the LOPs by using the intercept method (the tabular method) in example 3.

\begin{tabular}{|c|c|c|c|}
\hline Body & \multicolumn{3}{|c|}{ The three plotting elements of LOP } \\
\hline Alkaid & $A P$ & $\begin{array}{l}42^{\circ} \mathrm{N} \\
017^{\circ} 14.2^{\prime} \mathrm{W}\end{array}$ & $\begin{array}{c}Z n=047.9^{\circ} \\
a=10.4^{\prime} A w a y\end{array}$ \\
\hline Capella & $A P$ & $\begin{array}{l}42^{\circ} \mathrm{N} \\
017^{\circ} 24.8^{\prime} \mathrm{W}\end{array}$ & $\begin{array}{c}Z n=318.8^{\circ} \\
a=24.2^{\prime} \text { Away }\end{array}$ \\
\hline
\end{tabular}

Source: [5] Dutton's Nautical Navigation, pp. 332-335 (2004).

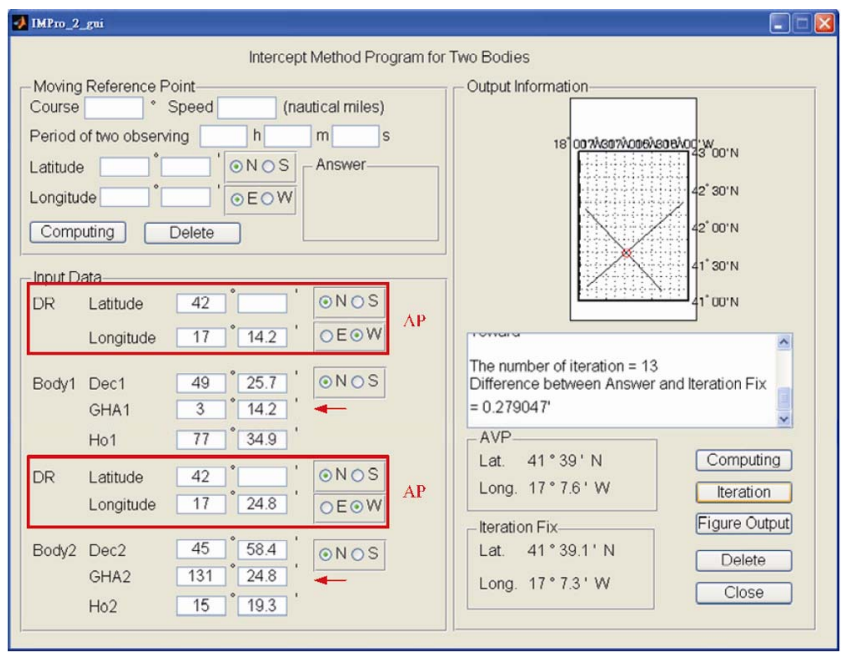

Fig. 8. Treating AP to run IMPro-2 for solving the AVP in example 3.

IMPro-2, the post-iteration AVP always is $L 41^{\circ} 39.1^{\prime} N, \lambda$ $017^{\circ} 07.3^{\prime} W$, as shown in Figs. 8 and 9, further demonstrating the accuracy of AVP. Besides, IMPro-2 can also demonstrate the manual plotting process; the figure in the upper-right part of this GUI, as shown in Figs. 8 and 9, can be expanded, outputted, and saved.

\section{CONCLUSIONS}

This paper proposes two computerized programs, ObsAltPro and IMPro-2, allowing users to easily and quickly complete computations of the AVP. Actually, the computerized solution is always more it avoids than tabular methods because it is free of rounding error. The above two programs, through demonstrated examples, have shown advantages of being simple, fast, and accurate. In summary, the characteristics of these two computation programs as are follows:

- Running ObsAltPro does not require information of nautical almanac at all, thereby it avoids its limitations and errors. The observed altitudes of various celestial bodies from 1986 to 2050 can be directly obtained.

- Due to the inherent nature of the IM, a trial-and-error method, and improving drawbacks of the IM. The developed

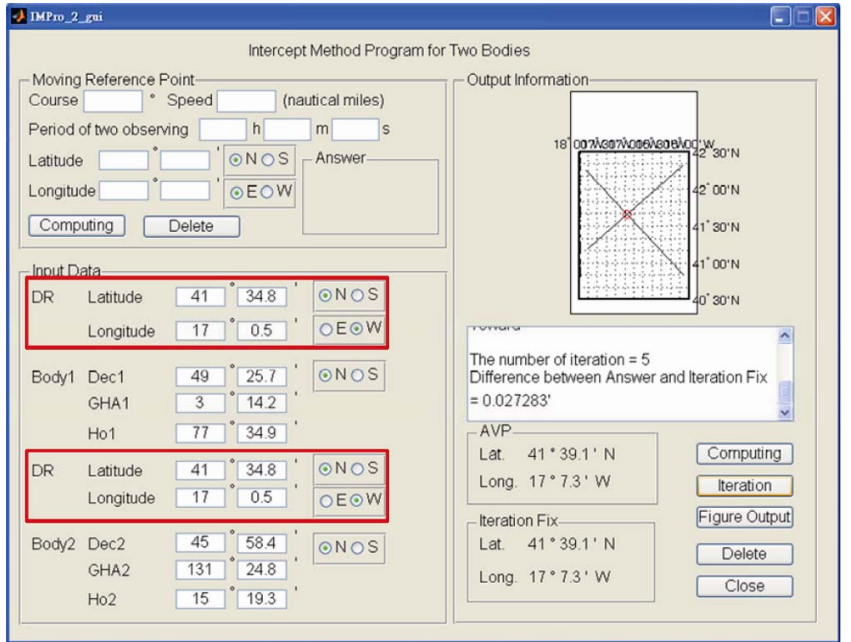

Fig. 9. Treating DR to run IMPro-2 for solving the AVP in example 3.

IMPro-2 not only directly uses the DR as the input variable to replace the AP but also adds an iteration function to verify the determined AVP.

- For complete solving the classical problem of celestial navigation, the moving reference point function should be taken into account. The developed IMPro-2 adopted rhumb line sailing in conjunction with parallel sailing to deal with this problem. Again, for conventional user, the developed IMPro-2 can also demonstrate the plotting figure to help illustrate the result.

\section{ACKNOWLEDGMENTS}

The first author would like to thank the National Science Council, Taiwan for financial support under contract number: NSC 96-2628-E-019-022-MY3.

\section{REFERENCES}

1. Bowditch, N., The American Practical Navigator, 2002 Bicentennial Ed., National Imagery and Mapping Agency, Bethesda, Maryland, U.S.A. (2002).

2. Bretagnon, P. and Francou, G., "Planetray theoris in rectangular and spherical variables. VSOP 87 solutions," Astronomy and Astrophysics, Vol. 202, pp. 309-315 (1988).

3. Chapront-Touzé, M. and Chapront, J., "The lunar ephemeris ELP 2000," Astronomy and Astrophysics, Vol. 124, pp. 50-62 (1983).

4. Clough-Smith, J. H., An Introduction to Spherical Trigonometry, Brown, Son \& Ferguson Ltd, Glasgow, Scotland, U.K. (1966).

5. Cutler, T. J., Dutton's Nautical Navigation, 15th Ed., Naval Institute Press, Annapolis, Maryland, U.S.A. (2004).

6. Her Majesty's Nautical Almanac Office, Rutherford Appleton Laboratory and the Nautical Almanac Office of the US Naval Observatory, 2008 Nautical Almanac, Commercial Ed., Paradise Cay Publications and Celestaire Inc., Taunton, Somerset, U.K. (2007).

7. Hohenkerk, C. Y. and Yallop, B. D., NavPac, Version 2.1.2, Council for the Central Laboratory of the Research Councils, Taunton, Somerset, U.K (2004).

8. Hsu, T. P., Chen, C. L., and Chang, J. R., "New computational methods for solving problems of the astronomical vessel position," The Journal of 
Navigation, Vol. 58, No. 2, pp. 315-335 (2005).

9. Maritime Safety Information Center, Sight Reduction Tables for Marine Navigation, Pub. No. 229, National Imagery and Mapping Agency, Bethesda, Maryland, U.S.A. (1981).

10. Meeus, J., Astronomical Algorithm, Willmann-Bell, Richmond, Virginia, U.S.A. (1998).

11. National Geospatial-Intelligence Agency, WGS 84 Earth Gravitational Model, (http://earth-info.nga.mil/GandG/wgs84/gravitymod/egm2008/ egm08_wgs84.html) Retrieved Jul. 9th, 2009.

12. Reis, O., Navigator, Version 4.0, Celestaire, Wichita, Kansas, U.S.A. (2005).

13. STARPILOT, LLC, STARPILOT PC, Maryville, Tennessee, U.S.A. (2002).

14. Van Allen, J. A., "An analytical solution of the two star sight problem of celestial navigation," NAVIGATION, Journal of Institute of Navigation, Vol. 28, No. 1, pp. 40-43 (1981). 